\title{
Visiones del alumnado y el sentido del prácticum en educación primaria
}

\author{
Pablo CORTÉS-GONZÁLEZ \\ Blas GONZÁLEZ ALBA \\ Daniela PADUA ARCOS
}

\begin{abstract}
Datos de contacto:
Pablo Cortés-González Universidad de Málaga pcortes@uma.es

Blas González Alba Universidad de Málaga blas@uma.es

Daniela Padua Arcos Universidad de Almería dpadua@ual.es
\end{abstract}

\section{RESUMEN}

El presente trabajo recoge el proceso de análisis y reflexión compartida en torno a cómo contribuye el periodo de prácticas en la formación académica y en la identidad profesional de los estudiantes del grado de primaria de las universidades de Málaga y Almería. Durante los cursos 2016/17, 2017/18 y 2018/19 y en el marco del Proyecto de Investigación denominado 'La identidad profesional del profesorado novel', se han investigado con un total de 192 alumnos de prácticas de ambas universidades. A partir de grupos de reflexión y discusión, entrevistas y las valoraciones que el alumnado ha plasmado en sus porfolios, se hace un acercamiento a sus respectivas experiencias en la asignatura del Prácticum. Sus voces nos muestran la distancia percibida y existente entre el currículum expuesto en el aula universitaria y la realidad escolar, y el impacto experimentado por el alumnado tras comprobar que muchos centros de prácticas no han cubierto sus expectativas. Este proceso compartido, colectivo y reflexivo desarrollado en la asignatura del Prácticum y el proceso de la investigación ha permitido al alumnado reconstruir y deconstruir su identidad docente y mostrar algunas de las debilidades y limitaciones percibidas en cuanto a la relación universidad-centro de prácticas.

PALABRAS CLAVE: Prácticum, educación primaria, currículum, escuela, identidad docente 


\title{
Students' perspectives and the meaning of the practicum in primary education
}

\begin{abstract}
This paper collects the process of analysis and shared reflection about how the prácticum period contributes in the academic training and in the professional identity of the primary degree of the universities of Malaga and Almería' students. During the 2016/17, 2017/18 and 2018/19 academic years, and within the framework of the Research Project called 'The professional identity of new teachers', a total of 192 prácticum students from both universities participated. From reflection and discussion groups, interviews and the evaluations that the students have reflected in their porfolios, we approach their respective experiences in the subject of the prácticum. Their voices show us the perceived and existing distance between the curriculum exposed in the university classroom and the school reality, and the impact experienced by the students after verifying that many schools have not met their expectations. This shared, collective and reflective process developed in the prácticum subject and the research process has allowed students to reconstruct and deconstruct their teaching identity and show some of the perceived weaknesses and limitations regarding the university-school relationship.
\end{abstract}

KEYWORDS: Practicum, primary education, curriculum, school, teaching identity

\section{Introducción}

El prácticum de maestros y maestras es un periodo formativo imprescindible en la formación del profesorado que, grosso modo, permite al alumnado ver, hacer, ver hacer $y$ hacer ver (Gonzáles, 2001), desarrollar competencias específicas y transversales (Coiduras, Gervais y Correa, 2009) y consolidar aprendizajes relevantes para su desarrollo profesional (Egido y López, 2016). Es decir, se trata de una práctica educativa que favorece la formación integral y extensa del alumnado (Carless y Prodan, 2003) más allá de las fronteras del aula universitaria.

Siendo conocedores de la importancia que el prácticum tiene para el alumnado tanto en el ámbito personal como profesional, presentamos los resultados de un trabajo de análisis y reflexión compartida en torno a cómo contribuye el periodo de prácticas en la formación educativa y en la identidad profesional de los estudiantes del grado de primaria, así como algunas debilidades y potencialidades percibidas sobre el mismo. Nuestra experiencia 
docente se circunscribe a las universidades de Málaga y Almería.

El prácticum en Educación Primaria en los planes de estudio de ambas instituciones se estructura en cuatro espacios temporales y en un total de 44 ECTS, coincidentes en la propuesta curricular universitaria en los cursos de segundo, tercero y cuarto de grado. Sobre estas últimas, se divide en dos partes: una primera etapa (que acoge los tres primeros periodos de prácticas) en la que desarrollan prácticas genéricas y, otra posterior que coincide con sus respectivas especialidades -menciones-. En relación a esto último, este periodo corresponde con el Prácticum III.2, y con respecto a las menciones el alumnado puede elegir entre: Educación física, Educación Musical, Escuela Inclusiva y Atención a la Diversidad, Francés, Genérico e Inglés.

Los centros de prácticas que se ofertan en los mencionados planes de estudio pasan por clasificarse, salvo algunas excepciones, entre los siguientes tipos de centros educativos de educación primaria (tanto públicos -la mayoria-, como concertados):

- Escuelas de educación primaria genéricas.

- Escuelas con proyectos ANAE (Atención a la Necesidades Específicas de Apoyo Educativo).

- Escuelas de educaciones específicas: centros bilingües, centros de educación especial, rurales.

- Escuelas comunidad de aprendizaje (de acuerdo con la Orden de 8 de junio de 2012, por la que se regula el procedimiento de inscripción y continuidad de centros reconocidos como "Comunidad de Aprendizaje" y se crea la Red Andaluza "Comunidades de Aprendizaje").

La propuesta que se hace desde las guías docentes y en el planteamiento del prácticum en los nuevos planes de estudio de la universidad española, suponen una posibilidad para la mejora formativa del alumnado (Moril, Ballester y Martínez, 2012). Concretamente, entre otros aspectos, se espera que el alumnado transite desde el primer año de la observación a la intervención educativa; o bien, a una experiencia investigadora. Son cuestiones que se han ido avanzando en los nuevos planes de la enseñanza universitaria española, aunque aún existen muchas incertidumbres al respecto (Latorre y Blanco, 2011).

Al respecto, pensamos que hay bastante flexibilidad normativa para que el alumnado, de acuerdo con las demandas de su tutor 
de prácticas y del centro escolar, pueda tener una experiencia amplia de la acción docente. Lo que se intenta es permitir o dotar al alumnado de la posibilidad de actuar activamente en los centros, entendiendo que de esta forma se contribuye de manera más positiva a su desarrollo profesional y aprendizaje en el campo de la educación.

No obstante, a pesar de que hay un planteamiento cada vez más 'educativo' de lo que deben ser las prácticas, aún encontramos bastantes escollos que dificultan lo que se debería esperar de la formación universitaria en educación; y de alguna manera, influye frontalmente en la construcción de la identidad del alumnado de los estudios de Educación primaria y, añadimos, de todos los agentes involucrados en la formación de maestros.

\section{Marco teórico}

Cualquier proceso formativo, más allá de la transformación personal y profesional que pueda desencadenar en el alumnado, tiene como principal objetivo posibilitar que los estudiantes integren los conocimientos y sean capaces de emplearlos en su práctica profesional (Correa, 2015).

En el marco de la formación del profesorado a este periodo, obligatorio para la obtención del título y regulado, se le denomina "prácticum" y supone un aprendizaje por inmersión (Armengol, Castro, Jariot, Massot y Sala, 2011) que posibilita al alumnado entrar en contacto, desde la posición de "docente en prácticas", con la realidad educativa y escolar (Cid y Ocampo, 2006).

Esta asignatura tiene como principal objetivo potenciar la reflexión y la indagación sistemática y colaborativa (Susinos y Sáiz, 2016) a partir de promover aprendizajes y el desarrollo competencial en contextos reales. Por este motivo, el prácticum es una materia crucial en la formación del profesorado (González y Fuentes, 2011) que permite "participar activamente y conectar teoría y práctica a través de la reflexión en la acción para poder adquirir e interiorizar los conocimientos, destrezas, habilidades y actitudes propias de la profesión para la que se está formando" (Rodriguez-Hidalgo, Calmaestra-Villén y Maestre-Espejo, 2015, p.415) y aplicarlos en un contexto real. Es decir, facilita la integración y vinculación de los contenidos asimilados en la 
práctica y posibilita que se produzca conocimiento de la enseñanza, para la enseñanza y en la enseñanza (González y Fuentes, 2011).

Como señalan Cid y Ocampo (2006) "el Prácticum es una materia troncal cuyo desarrollo se realiza, básicamente, en contextos educativos ajenos a la institución universitaria, por lo que supone un asunto de relaciones institucionales ciertamente complejo y que exige el adecuado soporte normativo" (p.452), lo que conlleva que alumnado en prácticas, profesorado tutor profesional -centro educativo- y profesorado tutor -universidadasuman y contraigan una serie de obligaciones $y$ responsabilidades.

Desde una perspectiva formal y organizativa docente, el desarrollo del prácticum requiere de la presencia y del trabajo conjunto de:

- un tutor en el centro de trabajo o tutor profesional, responsable del proceso de aprendizaje del alumnado dentro del aula escolar, principal figura profesional para los y las estudiantes en prácticas (Puig, 2006) y comprometido de la evaluación del alumnado en prácticas en el contexto del aula (Martínez y Raposo, 2011).

- y de un tutor académico -profesorado vinculado a la universidad- (Villa y Poblete, 2004) que guía y asesora a los estudiantes en lo relativo a las dificultades y necesidades académicas, personales y/o profesionales que puedan encontrarse a lo largo del proceso formativo de prácticas. Asimismo, facilita que el alumnado vincule los conocimientos adquiridos en otras materias con las situaciones experimentadas en el aula.

Desde una dimensión formativa discente, el periodo de prácticas permite al alumnado analizar y reflexionar acerca de sus conocimientos (González y Fuentes, 2011) y poner en práctica competencias que no han sido posible desarrollar en la formación universitaria y que son exigidas para el ejercicio de la docencia. Como señalan Pascual, Ponce de León, Goicoechea, Torroba, y Fernández (2010) el alumnado en prácticas experimenta un proceso formativo caracterizado por la observación, la colaboración, la interacción e intervención en el aula, la planificación y la puesta en práctica de proyectos educativos. 


\section{Investigaciones sobre el alumnado en el prácticum}

Desde un plano investigador, no hemos encontrado muchos estudios que rescaten las voces del alumnado sobre su experiencia en el prácticum, pero sí que hay aportaciones de gran interés que lo sitúa como un campo necesario por el que seguir apostando. Destacamos tres ejes temáticos sobre los que se circunscriben la mayor parte de las aportaciones: (1) Análisis de competencias y tareas; (2) propuestas didácticas para el aprendizaje; (3) indagaciones sobre la identidad y sentido del prácticum.

Sobre el primer eje, cabe mencionar los trabajos de González, Fuentes y Rasposo (2006) y de Mendoza y Covarrubias (2014), que profundizan en las tareas y competencias que aportan el prácticum al alumnado, destacando la construcción de una visión crítica de la realidad educativa y la importancia de indagar desde la propia práctica.

Sobre el segundo eje, que es el más prolifero, abordan estrategias didácticas que aportan y mejoran los aprendizajes a partir del periodo de prácticas tales como la elaboración de blogs (Fernandez, Bartolome y Cano, 2013); la creación de diarios online (Llopis, 2017); o la mensajería instantánea como facilitadora de debates colectivos (Martín y Trigueros, 2016)

Si nos adentramos en el tercer eje, cabe destacar los trabajos de Calvo y Leite (2017) y Bedacarratx (2012), que expresan la importancia del prácticum y de la formación inicial en la identidad profesional docente. Ambos trabajos demandan periodos de prácticas más inmersos en la cotidianeidad de la escuela y de la universidad para materializar propuestas de transformación y colaboración entre los distintos agentes educativos.

\section{Metodología}

La presente investigación se enmarca en el paradigma interpretativo (Sandin, 2003); desde esta perspectiva pretendemos acercarnos a "las percepciones de los actores desde dentro, siendo una tarea fundamental la de explicar las formas en que las personas en situaciones particulares, comprenden, narran, 
actúan y manejan sus situaciones cotidianas" (Huberman y Miles, 1994, p. 5), es decir, conocer cómo los estudiantes de magisterio interpretan lo que aprenden y experimentan durante su periodo de prácticas y qué valor le dan a su identidad docente en el marco del prácticum.

La información ha sido recogida estos tres últimos años a través de entrevistas, grupos de reflexión y discusión con el alumnado (Porto y Ruiz, 2014), análisis documental de los portafolios (Jarauta y Bozu, 2013) y anotaciones que como tutores académicos hemos ido elaborando. Esta recogida de información es, en parte, fruto de los resultados del Proyecto de Investigación subvencionado con los fondos propios de la UMA denominado ' $\mathrm{La}$ identidad profesional del profesorado novel', en el que se rescata la mirada de estudiantes de primer y cuarto curso y profesorado novel (según la literatura al respecto, maestros y maestras de hasta cinco años de ejercicio docente), sobre la construcción de su identidad docente (Cortés, Leite, Rivas, 2014).

Sobre lo que respecta a esta aportación, el objetivo general se centra en analizar cómo contribuye el prácticum en la formación e identidad profesional del alumnado del grado de primaria. Respecto a los objetivos específicos, podemos decir:

- Reflexionar sobre el sentido del aprendizaje en la Universidad.

- Pensar cómo influye los aprendizajes en el grado en relación con los aprendizajes en los centros de prácticas

- Analizar fortalezas y debilidades (valoración) de las experiencias del prácticum, desde la voz del alumnado.

A lo largo de los cursos escolares 2016/17, 2017/18 y $2018 / 19$, se han tutorizado un total de 143 alumnos y alumnas de la Universidad de Málaga y 49 de la Universidad de Almería, lo que ha permitido realizar un total de 32 entrevistas individualizadas y 5 grupos de discusión (tres y dos respectivamente), por curso y grupo, como una estrategia que ha permitido que el alumnado comparta en el aula universitaria las reflexiones, limitaciones, potencialidades y aprendizajes derivados de sus respectivas experiencias.

Ejemplificando sobre el diseño de los instrumentos utilizados, cabe decir que las entrevistas fueron semi-abiertas a partir de cinco focos que emergieron de un primer análisis de los portafolios 
recogidos. Algunos ejemplos: ¿qué papel tiene las prácticas en tu formación docente?; ¿qué aspectos mejorarias y por qué?; ¿hay conexión entre los aprendizajes de las prácticas con el resto de las materias?, en caso afirmativo, ¿puedes profundizar en qué aspectos?... Referente a los grupos de discusión, una vez tuvimos un marco categórico con diversas aristas, nos dispusimos a diseñar pocas preguntas y comenzar con afirmaciones que alentasen al debate, la interacción y la reflexión conjunta. Algunos ejemplos que podemos destacar son: con las prácticas verdaderamente he aprendido a ser maestro; la formación teórica es necesaria para la práctica; el modelo formativo compagina correctamente los periodos de prácticas en los planes de estudio. Las preguntas se avanzaron una vez el debate se abría y dependiendo del hilo discursivo del grupo de reflexión. Algunas preguntas fueron: ¿qué propuestas harias para una mejora de los planes formativos?, ¿cómo generar estrategias que permitan tener más presencia en la facultad de las escuelas y viceversa?

La selección de los estudiantes se ha llevado a cabo mediante muestreo voluntario (Quintana, 2006) en tres instancias: (1) petición de participación a través de la recepción de portafolios para su análisis al total de estudiantes matriculados; (2) de estos estudiantes, anualmente se fue pidiendo y realizando la participación para la fase de entrevistas, en la que se respetó la proporcionalidad entre hombres y mujeres respecto a los matriculados en el grado $(66,5 \%$ mujeres y $33,50 \%$ hombres en el caso de la UMA y $61 \%$ de mujeres y $39 \%$ de hombres en la UAL); y (3) con esos 32 estudiantes, se realizaron grupos de reflexión en grupos de entre 6 y 8 participantes, organizados por criterios de diversidad en las respuestas obtenidas en las entrevistas y teniendo en cuenta la proporcionalidad de hombres y mujeres antes mencionada.

El proceso analitico se ha desarrollado en dos fases, una de "Codificación Abierta" y otra de "Codificación Axial" (Strauss y Corbin, 2002). Durante la primera etapa se han seleccionado y agrupado todas aquellas evidencias que nos han resultado clave para entender precisamente las contradicciones y reflexiones experimentadas por el alumnado durante su periodo de prácticas. En una segunda fase, y tras un proceso analítico más profundo, se han agrupado las evidencias en categorías comunes más 
depuradas, lo que ha posibilitado que emerjan los dos ejes analiticos señalados anteriormente.

Este proceso ha articulado nuestro trabajo en dos categorias que giran en torno a los contrastes, reflexiones y contradicciones formativas que el periodo de prácticas ha generado en el alumnado: (1) Separación entre las materias teóricas y el prácticum y (2) las expectativas y demandas sobre las prácticas como oferta universitaria-.

\section{Resultados y discusión}

Pensamos que las prácticas en la formación de los futuros maestros es una pieza clave dentro de todo el engranaje formativo y educativo, ya que supone una manera de aplicar los conocimientos y experiencia a la realidad escolar. Estos no solo se circunscriben a los adquiridos por parte del alumnado dentro de las aulas universitarias, sino también al profundo conocimiento sedimentado (Rivas, Cortés y Márquez, 2018) que traen consigo de la práctica escolar experimentada como alumnado en las aulas de educación infantil, primaria, secundaria y superior.

Nos referimos a que la experiencia escolar de los estudiantes de grado sobre la práctica escolar es elevada, pues han pasado unos 15 años de media de escolarización como estudiantes en escuelas de distintos niveles y características, y desde entonces, han ido interiorizando modelos docentes que van configurando su práctica educativa. Este hecho repercute en la construcción de una identidad docente particular, aún más teniendo en cuenta que es alumnado que ha optado por cursar estudios de educación.

Al respecto, entendemos que el prácticum no se constituye como la primera experiencia escolar ni educativa del alumnado, aunque sí evidenciamos que influye notablemente en el cambio de perspectiva que les ofrece estar 'encima de la tarima'. Esto último, por la información recogida, está estrechamente ligado a la situación de responsabilidad que asumen en las prácticas escolares.

La Figura I ofrece una panorámica en cuanto a la estructuración de las dos categorias mencionadas junto a los temas que las definen; asimismo, se ofrece una visual sobre la cantidad de registros/evidencias recogidos de cada uno de los instrumentos utilizados. Por la gran cantidad de información, 
hemos seleccionado fragmentos representativos a las aportaciones que se han ido manejando.

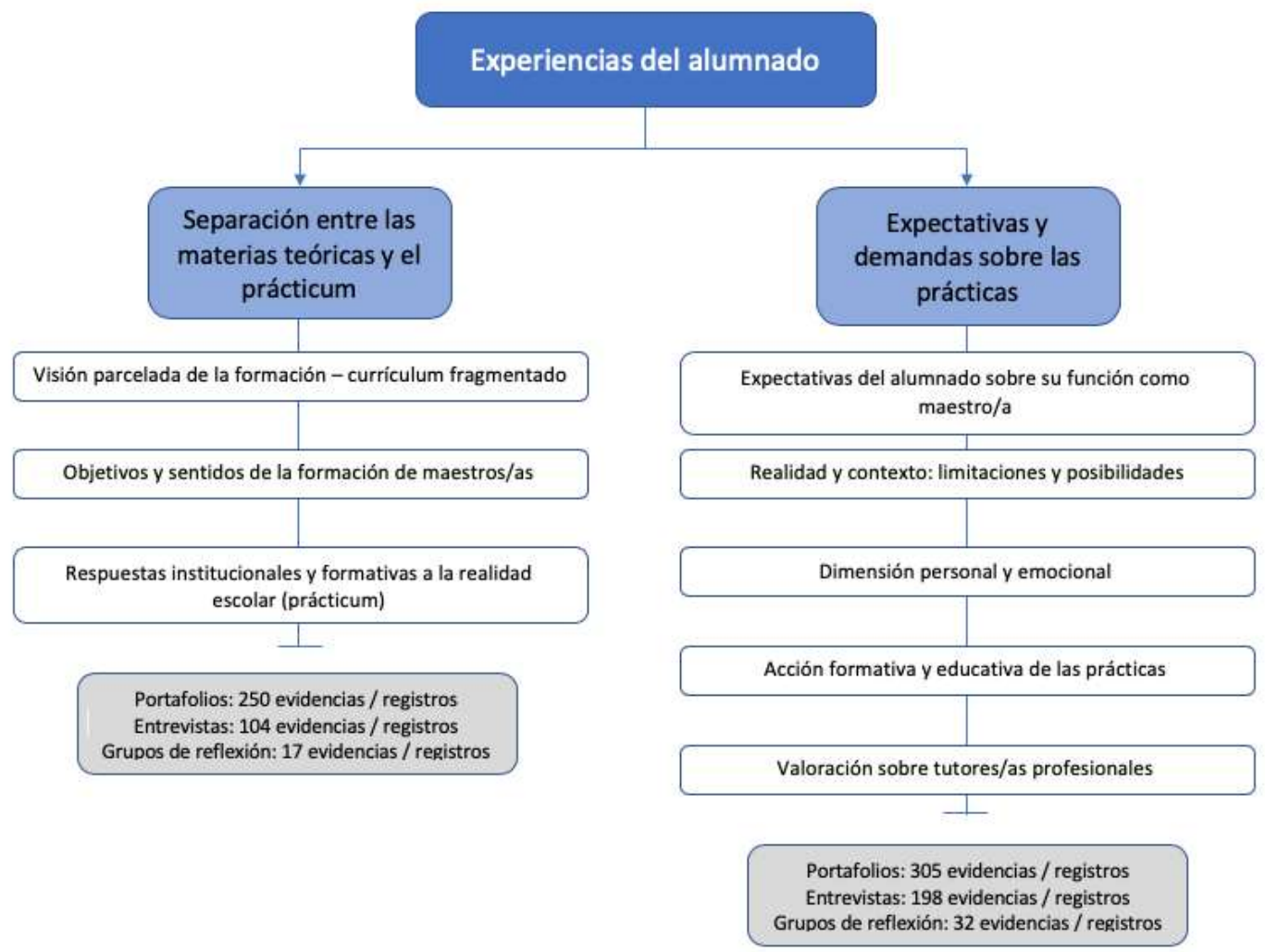

Figura 1. Cuadro de categorías interpretativas y relación de evidencias obtenidas.

\section{Separación entre las materias teóricas y el prácticum}

Esta cuestión está relacionada con lo que hemos apuntado anteriormente sobre la fragmentación de los currículos universitarios o lo que hemos denominado en otros trabajos como el conocimiento sedimentado (Rivas, Leite y Cortés, 2011; Rivas, Cortés y Márquez, 2018). El alumnado percibe que cada materia, a pesar de las muchas coincidencias temáticas y/o competenciales, tiene una definición parcelada respecto a todo el diseño formativo del currículum del grado de Educación Primaria (metodología, objetivos, evaluación...). Esta percepción 
imposibilita que se aprecie una perspectiva educativa global, aplicada, interrelacionada, significativa y crítica en el proceso formativo (Llopis, 2017).

El periodo de prácticas no deja de ser una materia más que refleja la distancia existente entre el currículum expuesto en el aula universitaria y la realidad escolar.

Estudiamos cosas que después en la realidad (escolar), es completamente distinta. Nos exigen cosas que no nos sirven o no le damos valor (...) mucha teoría que después no sabemos llevarla a la práctica. Y también en los centros vemos cosas que tenemos que aprender sobre la marcha (estudiante, grupo de discusión, 2016-2017).

Quitando algunas asignaturas y profesores, es en el prácticum donde de verdad se aprenden cosas de la vida real. Es verdad que he tenido mejores y peores experiencias, pero hay mucho que hacer en las escuelas (estudiante, entrevista, 2018-2019)

Encontramos sentido a algunos de los argumentos que apunta el alumnado, sobre todo en lo relativo a qué se estudia y para qué en la universidad. Estas son cuestiones centrales que nos interpelan, como docentes universitarios, a repensar nuestras actuaciones para con un alumnado que, en un breve periodo de tiempo, tendrá que afrontar situaciones escolares para la que, quizás, en la propia universidad no se ha dedicado suficiente tiempo ni se le ha dado la necesaria importancia.

Al respecto, una estudiante dice: "nos piden normas APA, nos machacan vaya, y luego en los coles no se ve eso" (estudiante educación primaria, entrevista, 2016-2017). Si solo nos centramos en la importancia del formato (como puede ser la aplicación de una normativa de citación) y olvidamos la importancia de la escritura como forma de intercambio de conocimiento y saberes, esta práctica carecería de sentido y distanciaria mucho más la necesidad de entender un enfoque práxico de la formación universitaria. Debemos de preguntarnos en qué medida la citación (el formato) debe estar por encima del interés o la preocupación por la capacidad crítica, la búsqueda de información y triangulación... y el desarrollo de competencias necesarias para actuar en un aula de primaria. 
Así mismo, nos preguntamos ¿en qué medida desde la universidad generamos contextos educativos y conocimientos que responden a las diversas realidades y situaciones de aula que el alumnado puede encontrarse durante el periodo de prácticas?; y esto pasa por cuestionarse el sentido de la formación universitaria, de la tradición tecnocrática de la enseñanza universitaria, la fragmentación disciplinar (Rivas, Cortés y Márquez, 2018; Rivas, Leite, Cortés, 2017) y la relación universidad-escuela (Bretones, 2013)

Mi sensación es que estamos con las asignaturas, termina un cuatrimestre o a mitad de otro, comenzamos las prácticas y no se relacionan con lo que estamos dando. Mi valoración como propuesta en este portafolio es que se podría unificar o hacer asignaturas puente con los prácticums (Estudiante, portafolios, curso 2017-2018)

Desde nuestro prisma, planteamos que las clases magistrales (bien dosificadas), deben conciliar con estrategias como el trabajo grupal, la realización de seminarios, el trabajo autónomo, las salidas a centros escolares, el voluntariado, las sesiones de mentoring con el profesorado..., en definitiva, con actuaciones educativas que acerquen al alumnado a las aulas escolares y a la creación de espacios y tiempos que permitan que esas experiencias sean compartidas, discutidas y reflexionadas en las aulas universitarias. De este modo, los periodos de prácticas serian parte de una experiencia de construcción, deconstrucción y reconstrucción del sentido de escuela, profesión e identidad docente.

En este sentido, de acuerdo con Martínez (2013), y a pesar de que en el ámbito de la formación del profesorado se han realizado ciertas modificaciones a nivel formal, precisamos de una transformación educativa que requiere de abrir procesos de reflexión acerca de las necesidades formativas reales del alumnado y de las limitaciones de algunas asignaturas para ajustarse a la realidad escolar, dimensiones que en gran medida quedan reflejadas en las valoraciones y reflexiones que manifiesta el alumnado en su portafolio y/o que expresan en las entrevistas y grupos de discusión. 


\section{Expectativas y demandas sobre las prácticas -como oferta universitaria-.}

La segunda cuestión planteada tiene que ver con las expectativas que el alumnado ha depositado en los periodos de prácticas. Principalmente este hecho se recalca en el caso del primer periodo de prácticas, donde apenas los estudiantes permanecen tres semanas en los centros y en el cual no da tiempo material para que se lleven una visión más o menos completa de la realidad educativa y particular a la que asiste.

En general he tenido una buena experiencia en el cole. Ha sido muy gratificante enfrentarme a la realidad que en el futuro quiero trabajar. Sé que más adelante las prácticas son más largas, pero en este prácticum I, cuando ya empiezas a adaptarte y conocer a los niños, los profes y el cole, nos tenemos que marchar (estudiante, entrevista, 2018-2019)

Así mismo, y durante otros periodos de prácticas más extensos $\mathrm{y}$ salvando algunas grandes excepciones, coincidiendo con el trabajo de Tejada y Carvalho (2013), el alumnado ha experimentado un prácticum caracterizado por un trabajo de aula fuertemente dependiente, asistente (hacia el tutor profesional) y limitado, que provoca que el periodo de prácticas se caracterice por observar y escuchar al profesor/a y desarrollar alguna tarea o actividad puntual con el alumnado (Barquín, 2002). Según las palabras de dos estudiantes:

Muchas veces los tutores de los centros están casi obligados; en el sentido que en muchas ocasiones les supone tiempo tener que coordinar a los estudiantes de la universidad y eso hace que no quieran salirse de sus tareas. A veces lo entiendo porque he visto a mi tutora que no para y no sabe de dónde sacar el tiempo para atendernos como merecemos (estudiante 2016-2017, grupo de discusión).

Este periodo de prácticas me ha permitido darme cuenta y reafirmarme en lo que quiero ser: maestra. (...) pero también he podido ver lo que no quiero hacer. He podido dar mucho más, pero al no ser la responsable de la materia pues no ha podido ser. Gracias al cole he hecho muchas cosas, pero por otro lado, también me han limitado mucho (estudiante, portafolio, 2017-2018). 
En algunas ocasiones el alumnado llega a los centros de prácticas con unas intenciones y expectativas que después no se pueden cumplir, ya sea por motivos relacionados con las dinámicas o características del profesorado, del aula, del propio centro, del propio estudiante, o una combinación de algunas de ellas, tal y como también se expresa en Bretones (2013). Esta situación, que suele generar frustración en el alumnado, es el resultado de una construcción previa, normalmente idealizada de escuela, que vincula la función docente a la impartición de docencia y a los modelos hegemónicos de entender las funciones del docente.

Resulta interesante comprobar cómo el periodo de prácticas permite al alumnado confrontar su formación, experiencias, conocimiento, habilidades, competencias... con la realidad escolar experimentada. Este ejercicio de autorreflexión facilita la reconfiguración y deconstrucción de su identidad docente; el aula donde se desarrolla el prácticum es un espacio para comprender aplicando la teoría, para aprender completando la teoría y para aprender contrastando la teoría (González, 2001)

Las prácticas se me hacen cortas. Es en el momento en el que puedo sentirme como maestra. Me tiro muchas horas sentada en las sillas de la universidad y es una manera de entrar en el campo donde voy a trabajar en el futuro (...) hay cosas que me gustan y otras que me gustaria cambiar, pero de todo se aprende, ¿no? (Alumna educación primaria, grupo de discusión, 2017-2018).

Realizando este portafolios me he dado cuenta de la importancia que tiene todo el trabajo de análisis con mis profesores/as, especialmente con XXX. Siempre nos insiste que a teoria y la practica se necesitan, porque para poder comprender y hacer con sentido, hay que plantearse y darle muchas vueltas, leer lo que hacen otros, etc. (Alumno educación primaria, portafolios, 2016-2017).

En muchas ocasiones encontramos a algunos tutores profesionales de prácticas que por desconocimiento, falta de definición de sus funciones para con el alumnado en prácticas o bien por motivos personales y/o profesionales relacionados con su aula, el colegio o la burocracia, no pueden atender a los 
estudiantes en prácticas, o no lo hacen en el grado y modo que el alumnado espera.

Al principio fue muy desagradable, cuando mi tutor me decía que le quitaba tiempo y que no estaba para estas funciones. Al final, gracias a la PT pude engancharme. También la directora me ayudó mucho a poder hacer cosas, ya que estaba muy perdida (estudiante educación primaria, grupo de discusión, 2017-2018).

También, resulta interesante que el alumnado reflexione en su periodo de prácticas acerca de las distintas limitaciones relacionadas con los recursos -humanos y materiales- y con las diversas ecologías y culturas escolares (Martínez y Fernández, 2018) inherentes a cada uno de los centros escolares, y que en cierta medida provocan una confrontación con sus esquemas mentales y bajo los cuales se construye, reconstruye y deconstruye su identidad docente. Esta cuestión la observamos en bastantes registros, pero destacamos como lo expresa el siguiente estudiante:

Me cuesta mucho conectar la práctica con la teoría. Si es cierto que a medida que he ido avanzando en la formación me voy dando cuenta de esta importancia. A veces nos dejamos llevar, pero sin teoría, sin reflexión es dificil hacer propuestas potentes y valorar realmente las dificultades de la docencia. Otras veces, piensa que estoy trabajando educativamente y gracias a la teoria me doy cuenta de que a lo mejor he hecho una actividad que ha excluido a un alumno. Te abre los ojos. (Estudiante educación primaria, entrevista, 2018-2019).

Algunos estudiantes encuentran realidades ancladas en una visión de escuela tradicional, basada en el aprendizaje memorístico, repetitivo y jerarquizado, que, por un lado, tropieza diametralmente con otras propuestas innovadoras y/o transformadoras experimentadas previamente en el marco del aula universitaria, en un periodo de prácticas anterior u en otras experiencias escolares -voluntariado, maestros/as sombras...-: "Llevaba meses estudiando cosas, estoy de voluntario en una comunidad de aprendizaje y cuando he querido ir a otro centro me he encontrado con muchas limitaciones. En verdad, son por parte de las personas, que no quieren ver otras alternativas" (estudiante, entrevista, curso 2018-2019); en otras ocasiones, encuentran 
grandes limitaciones para desarrollar planteamientos alternativos o, simplemente, distintos: "cada docente está en su lugar y en su materia, es dificil aplicar lo que muchas veces aprendemos en clase" (estudiante, grupo de discusión, curso 2016-2017).

Esta cuestión mantiene una estrecha relación con las culturas y las ecologías docentes y de centro (Martinez y Fernández, 2018; Diez y Mallo, 2018). En algunas materias se hace hincapié, acertadamente, en la necesidad de hacer una revisión pedagógica de la actividad y metodología docente, de la innovación (Gutiérrez y Becerra, 2014) o de la transformación (Rivas, Leite y Cortés, 2017), de las prácticas docentes, no obstante, la mayor parte de centros continúan aferrados a modelos organizativos y metodologías más tradicionales. Este hecho provoca que el alumnado, que conoce estrategias y metodologías innovadoras y/o ha participado en experiencias de voluntariado, de aprendizaje servicio, en comunidades de aprendizaje... durante un periodo de prácticas anterior o como una actividad paralela a su formación, perciba que los planteamientos metodológicos y organizativos transformadores, que se potencian desde la formación universitaria, son incompatibles con la realidad de muchos de los centros educativos y escolares en los que han realizado sus periodos de prácticas.

\section{Conclusiones}

En este punto del análisis, concluimos con varias propuestas que sintetizamos fruto del trabajo de investigación y que se posicionan como debilidades del proceso de prácticas experimentado por el alumnado:

- Necesidad de mayor cohesión y coordinación entre la universidad y los centros escolares. Los periodos de prácticas juegan un papel fundamental ya que aúnan alumnado, profesorado y tutores profesionales en un mismo marco de relaciones. Dotar de mayor responsabilidad y participación en la gestación y gestión de dichos periodos a los agentes citados, facilitaría la cohesión e implicación (Bretones, 2013), mejorando las competencias profesionales del alumnado (Mendoza y Covarrubias, 2014). 
- Cultura de la incertidumbre. Las prácticas, en ocasiones, se entienden como una experiencia inconexa con lo aprendido en las materias de grado y la realidad experimentada. En este sentido, es necesario incentivar estructuras curriculares más abiertas y con visiones desde lo específico a lo global (Gutiérrez, Correa, Jiménez e Ibáñez, 2009; LLopis, 2017). Pensamos que una absoluta fragmentación provoca incertidumbre en los procesos formativos, romper con esta fragmentación no es sólo una cuestión de contenidos, sino, más bien, de entender la relación y el vinculo existente entre todas las materias que cursa el alumnado a lo largo de su formación superior, lo que facilitarian en nuestra opinión mayor entendimiento $\mathrm{y}$ coordinación entre profesorado universitario.

- Confirmar el interés por la educación. Hemos apuntado brevemente que la formación universitaria trasciende de lo formativo y/o profesional y que es una apuesta ética $\mathrm{y}$ política. Teniendo en cuenta esto, la educación se percibe como unos de los pilares para el entendimiento de los contextos sociales, políticos y culturales de la sociedad actual. Siendo, además, una cuestión que está íntimamente ligada a la construcción de la identidad docente: ¿por qué nos insertamos en el sistema educativo de manera profesional?, ¿qué principios y objetivos tengo?, ¿qué entiendo por educar?, ¿para qué?... (Cortés, Leite, Rivas, 2014)

- La formación universitaria desde un enfoque más práxico. Es decir, comprender qué tipo de conocimiento, saberes y experiencias entendemos como claves en la formación de maestros y maestras y cómo configuramos nuestra identidad profesional docente. No nos referimos a un enfoque fundamentado en la práctica, en detrimento de un entendimiento crítico y reflexivo de la realidad educativa, sino que planteamos una mirada que facilite al alumnado la capacidad de dialogar, contrastar, construir, de-construir... con otros relatos, experiencias y realidades a partir de su experiencia sobre lo educativo. En este sentido, encontramos experiencias en la literatura pedagógica (Gutiérrez, Correa, Jiménez e Ibáñez, 2009), sobre modelos reflexivos (Llopis, 2017) y narrativas en la formación de los docentes y los 
periodos de prácticas que promueven este planteamiento (Calvo y Leite, 2017).

Estos cuatro ejes nos permiten elaborar conclusiones sobre la experiencia de las prácticas en el grado de primaria, vinculados con otros planteamientos de otras investigaciones, como la 'Red de Escuelas Inclusivas' (Márquez, Ibáñez y Padua, 2014). Es interesante rescatar los principios educativos en los que se apoyó y que nos parecen interesantes reflejar en esta ocasión por el carácter rupturista de la propuesta y como excusa propositiva al respecto (pp. 71y ss.): innovación; inclusión; transformación de las personas que participan en el aula; democracia; y justicia social. Estos elementos los entendemos como ejes clave para la transformación del sentido del prácticum para una experiencia educativa más profunda y significativa.

Esto es, a partir de los resultados de este estudio y los planteamientos expuestos en el marco teórico, podemos afirmar que cuando el espacio curricular y académico, tanto dentro como fuera de las aulas, desde las materias más teóricas a las más prácticas, se abre a la experiencia compartida, al diálogo y a la cooperación, basada en una construcción de saberes ecológicos (Martínez y Fernández, 2018), la experiencia del prácticum se convierte en un espacio que sitúa al alumnado frente a una realidad compleja y dispar (según contextos, profesionales...), en la que debe atender y resolver situaciones constantemente a través de la indagación (Susinos y Sáiz, 2016).

Es por ello que debemos pensar la formación universitaria de maestros y maestras como una forma de construir o tejer la identidad profesional docente en diálogo con las tradiciones académicas, sociales, culturales, científicas y politicas (Leite, Cortés, Rivas, 2016), con el objeto de plantear nuevas formas y estrategias (González y Fuentes, 2011) para la escuela que tengan un fondo educativo ( $\mathrm{y}$ no meramente basado en los contenidos, por ejemplo).

Esto pasa, además, por revisar varios elementos claves en el contexto sociopolítico universitario, que debe seguir avanzando en la coordinación entre agentes (Villa y Poblete, 2004), de distintos sectores educativos, al menos, en lo que se refiere al ámbito educativo, y en la mejora de las relaciones y sinergias en materia de prácticas profesionales entre las facultades de educación y los 
centros educativos (Allen, Ambrosetti y Turner, 2013). En este punto, los periodos de prácticas cobran especial relevancia.

Dicho de otro modo, los roles de los agentes educativos aún siguen bastante estancos como observamos en Puig (2006); ante esto, debemos plantear la experiencia formativa del prácticum, no simplemente como una asignatura en la que el alumnado es receptor de 'saberes' prácticos dentro de un aula, a diferencia de lo que plantean Rodríguez-Hidalgo, Calmaestra-Villén y MaestreEspejo (2015), sino como una experiencia práxica de transformación, construcción, re-construcción y de-construcción de la identidad docente y de los conocimientos adquiridos (González, Leite y Rivas, 2018). Para ello, se debe habilitar espacios y tiempos que permitan al alumnado exponer $\mathrm{y}$ reflexionar acerca de sus respectivas experiencias de prácticas con el objetivo de utilizar este conocimiento para mejorar una asignatura que, sin duda, es clave en la formación docente.

\section{Referencias}

Allen, J., Ambrosetti, A., y Turner, D. (2013). How school and university supervising staff perceive the pre-service teacher education practicum: a comparative study. Australian Journal of Teacher Education, 38(4), 108-128. http://dx.doi.org/10.14221/ajte.2013v38n4.9

Armengol, C., Castro, D., Jariot, M., Massot, M., y Sala, J. (2011). E1 Prácticum en el Espacio Europeo de Educación Superior (EEES): Mapa de competencias del profesional de la educación. Revista de Educación, 354, 71-98.

Barquín, J. (2002). La tutorización de las prácticas y la socialización del futuro profesorado. Revista de Educación, 327, 267-283.

Bedacarratx, V. A. (2012). Futuros maestros y construcción de una identidad profesional: una mirada psicosocial a los procesos que se ponen en juego en los trayectos de formación en la práctica. REDIE. Revista Electrónica de Investigación Educativa, 14(2), 133-149

Bretones, A. (2013). El prácticum de magisterio en educación primaria: una mirada retrospectiva. Revista Complutense de 
Educación, 24(2), 443-471. https://doi.org/10.5209/rev_RCED.2013.v24.n2.42088

Calvo, P., y Leite, A. (2017). El triángulo de la tutorización: Alumnado, universidad y centros de prácticas. RIUMA. https: / / riuma.uma.es/xmlui/handle/10630/14193

Carless, S., y Prodan, O. (2003). The impact of Practicum training on career and job search attitudes of postgraduate psychology students. Australian Journal of Psychology, 55(2), 89-94. https: / /doi.org/10.1080/00049530412331312944

Carvalho, M. L., y Tejada. J. (2013). El prácticum en la formación inicial de maestros: percepciones de los tutores de universidad de educación infantil y primaria. En Actas XII Congreso Internacional sobre el prácticum, Un prácticum para la formación integral de los estudiantes, Andavira Editora

Cid, A., y Ocampo, C. (2006). Funciones tutoriales en el Prácticum correspondientes al actual plan de estudios de Magisterio de la Universidad de Vigo. Revista de Educación, 340, 445-472.

Coiduras, J., Gervais, C., y Correa, E. (2009). El contexto escolar como escenario de educación superior en la formación de docentes. El Prácticum en Quebec como modelo para reflexionar ante las nuevas titulaciones de grado. Educar, 44, 11-29. https: / / doi.org/10.5565/rev/educar.104

Correa, E. (2015). La alternancia en la formación inicial docente. Educar, $\quad 51(2)$,

259-275. http: / /doi.org/10.5565/rev/educar.712

Cortés, P., Leite, A., y Rivas, J.I. (2014). Un enfoque narrativo de la identidad profesional en profesorado novel. Tendencias Pedagógicas, 24, 199-214. http://doi.org/10.15366/tp

Diez, E., y Mallo, B. (2018). “Ecologías del aprendizaje invisible y ubicuo en el ámbito de la formación de formadores". En J.B. Martínez y E. Fernández. Ecologías del aprendizaje. Educación expandida en contextos múltiples. Madrid: Morata.

Egido, I., y López, E. (2016). Condicionantes de la conexión entre la teoría y la práctica en el Prácticum de Magisterio: Algunas evidencias a partir de TEDS-M. Estudios sobre Educación, 30, 217- 237. http:/ / doi.org/10.15581/004.30.217-237 
Fernández, M., Bartolomé, A., y Cano, E. (2013). Desarrollo y autoevaluación de competencias mediante blogs en el prácticum de Educación Primaria. En P. C. Muñoz, M. Raposo, M. González, M. E. Martínez, M. A. Zabalza, y A. Pérez (coord.). Un Prácticum para la formación integral de los estudiantes (pp. 699-712). Santiago: Andavira.

González, B., Leite, A. E., y Rivas, J. I. (2018). La indagación educativa como herramienta de transformación social. En M. T. Castilla y V. Martín Solbes (Coords.), Educación, derechos humanos y responsabilidad social (pp.127-142). Barcelona: Octaedro.

González, M. (2001). ¿Qué se aprende en el prácticum? ¿Qué hemos aprendido sobre el prácticum? En L. Iglesias et al. (Coords.), Desarrollo de competencias personales y profesionales en el Prácticum. VI Symposium Internacional sobre el Prácticum. Lugo: Unicopia

González, M., Fuentes, E. J., y Raposo, M. (2006). De alumno a profesor: análisis de las tareas realizadas durante las prácticas escolares. Revista Galego-Portuguesa de Psicoloxía e Educación, 11-12(13), 277-294.

González, M., y Fuentes, E. J. (2011). El Prácticum en el aprendizaje de la profesión de docente. Revista de Educación, 354, 47-70.

Gutiérrez, L. C., Correa, J. M. G., Jiménez, E. A., e Ibáñez, A. (2009). El modelo reflexivo en la formación de maestros y el pensamiento narrativo: Estudio de un caso de innovación educativa en el Prácticum de Magisterio. Revista de Educación, 350, 493-505.

Gutiérrez, P., y Becerra, M. T. (2014) Los Entornos Personales de Aprendizaje (PLE). Una experiencia de aprendizaje informal en la formación inicial del profesorado. RELATEC: Revista Latinoamericana de Tecnología Educativa, 13(2), 49-60. https: / /doi.org/10.17398/1695-288X.13.2.49

Huberman, M., y Miles, M. (1994). Manejo de datos y métodos de análisis. En N. K. Denzin y Y. S. Lincoln (Eds.), The Sage handbook of qualitative research (pp. 428- 444). California: Sage Publications. 
Jarauta, B., y Bozu, Z. (2013). Portafolio docente y formación pedagógica inicial del profesorado universitario: Un estudio cualitativo en la universidad de Barcelona. Educación XXI: Revista De La Facultad De Educación, 16(2), 343-361. https: / /doi.org/10.5944/educxx1.16.2.2646

Latorre, M. J., y Blanco, F. J. (2011). El prácticum como espacio de aprendizaje profesional para docentes en formación. Revista docente universitaria, 9(2), 35-54. https://doi.org/10.4995/redu.2011.6157

Leite, A., Cortés, P., y Rivas, J. I. (2016). Narrativa y creatividad en la Universidad: ¿es posible transitar otros caminos en la enseñanza y el aprendizaje? En P. Cortés-González y M. J. Márquez. Creatividad, Comunicación y Educación. Más allá de las fronteras del saber establecido (pp. 151-164). Málaga: UMA Editorial.

Llopis, M. A. (2017). Pensamiento reflexivo en el Prácticum I del alumnado de Grado de Maestro en Educación Primaria a través de diarios online. Universitat Jaume I. Tesis doctoral.

Márquez, M. J., Ibáñez, L., y Padua, D. (2014). Compromiso Socioeducativo y metodologias Inclusivas. En: A. Moreno, MArancibia (Eds.). Educación y Transformación Social: construyendo una ciudadanía crítica (pp. 207-229). Valparaíso: Ediciones Universitarias de Valparaíso.

Martín, J. M., y Trigueros, C. (2016). Mensajería instantánea y construcción compartida de significados: una experiencia de aprendizaje colaborativo en el Prácticum de Maestro de Educación Primaria. RED. Revista de Educación a Distancia, 51, art. 4.

Martínez, B. J. (2013). Las reformas en la formación inicial del profesorado. ¿Pero cuáles son los buenos saberes de las buenas maestras? Revista Interuniversitaria de Formación del Profesorado, 78(3), 89-102.

Martínez, E., y Raposo, M. (2011). Funciones generales de la tutoría en el prácticum: Entre la realidad y el deseo en el desempeño de la acción tutorial. Revista de Educación, 354, 155-181.

Martínez, J. B., y Fernández, E. (2018). Ecologías del aprendizaje. Educación expandida en contextos múltiples. Madrid: Morata. 
Mendoza, M., y Covarrubias, C. G. (2014). Competencias Profesionales Movilizadas en el Prácticum: Percepciones del Estudiantado del Grado de Maestro en Educación Primaria. Actualidades Investigativas en Educación, 14(3), 204-229

Moril, R., Ballester, L. y Martínez, J. (2012). Introducción de las matrices de valoración analítica en el proceso de evaluación del prácticum de los grados de infantil y de primaria. REDU Revista de Docencia Universitaria, 10(2), 251-271. https: / /doi.org/10.4995/redu.2012.6107

Pascual, T., Ponce de León, A., Goicoechea, M. A., Torroba, M. T., y Fernández, M. L. (2010). Prácticum: orientaciones para el plan de prácticas de la Diplomatura de Maestro. Logroño: Universidad de la Rioja.

Porto, L., y Ruiz, J. (2014). Los Grupos de discusión. En K. Sáenz y G. Tamez (Eds.), Métodos y Técnicas Cualitativas y Cuantitativas Aplicables a la Investigación en Ciencias Sociales (pp.253-273). México: Tirant Humanidades.

Puig, C. (2006). La profesionalización del estudiante y el espacio práctico de calidad. Plan de Prácticum de intervención e innovación docente: el rol docente del tutor de práctica. El acompañamiento del estudiante. Formación y supervisión. Acciones e investigaciones sociales, 1, 1-20.

Quintana Peña, A. (2006). Metodología de investigación científica cualitativa. En Quintana Peña, A., y Montgomery, W. (Eds.) Psicología tópicos de actualidad, (pp. 65-73). Lima: UNMSM.

Rivas, J. I., Cortés, P., y Márquez, M. J. (2018). Experiencia y contexto: formar para transformar. En C. Monge-López y P. Gómez (coords.). Innovando la docencia desde la formación del profesorado. Propuestas y realidades (pp. 109-124). Madrid: Sintesis.

Rivas, J. I., Leite, A., y Cortés, P. (2011). Luchando contra la historia: obstáculos para la innovación en la formación del profesorado, derivados de las historias escolares del alumnado. Educación y Pedagogía, 61, 69-79.

Rivas, J. I., Leite, A., y Cortés, P. (2017). Deconstruyendo las prácticas de formación: narrativa y conocimiento. En M. Pérez- 
Ferra y J. Rodríguez-Pulido. Buenas prácticas docentes del profesorado universitario (pp. 31-46). Barcelona: Octaedro.

Rodríguez-Hidalgo, A. J., Calmaestra-Villén, J., y Maestre-Espejo, M. (2015). Desarrollo de competencias en el prácticum de maestros: Abpy coaching multidimensional. Profesorado. Revista de Currículum y Formación de Profesorado, 19(1), 414434.

Sandín, M. P. (2003). Investigación Cualitativa en Educación: Fundamentos y Tradiciones. Madrid: McGraw Hill.

Strauss, A., y Corbin, J. (2002). Bases de la investigación cualitativa. Técnicas y procedimientos para desarrollar la teoría fundamentada. Bogotá: CONTUS

Susinos, R., y Sáiz (2016). Los problemas pedagógicos son mis aliados. El Prácticum como un proceso de reflexión e indagación colaborativa. Revista de Investigación en Educación, 1(14), 5-13.

Villa, A., y Poblete, M. (2004). Prácticum y evaluación de competencias. Profesorado. Revista de Currículum y Formación del Profesorado, 8(2), 1-19. 